\title{
Effect of Monthly Evaluations on Irrational Rates of Antimicrobial Drug Prescription in Outpatient and Emergency Departments
}

\section{Qiong Yang}

Ningbo Ningbo Sixth Hospital

Fangfang Yuan

Ningbo Ningbo Sixth Hospital

Li Li

Ningbo Ningbo Sixth Hospital

Jianfeng Jin

Ningbo Ningbo Sixth Hospital

Junhong He ( $\nabla$ 2063940547@qq.com )

Ningbo Ningbo Sixth Hospital

\section{Research Article}

Keywords: Antibiotic stewardship, antibiotic usage, antibiotic resistance, antibiotic prescription rate, monthly evaluation, retrospective study

Posted Date: November 30th, 2021

DOI: https://doi.org/10.21203/rs.3.rs-1048894/v1

License: (c) (i) This work is licensed under a Creative Commons Attribution 4.0 International License. Read Full License 


\section{Abstract}

Reduction of the excessive rate of antibiotic prescription is needed to curb antibiotic resistance. This retrospective study was conducted to verify whether monthly evaluations of antibiotic prescriptions could improve clinical antibiotic use in outpatient and emergency departments. Every month, from July 2016 to June $2019,25 \%$ of the antibacterial prescriptions from the outpatient and emergency departments in our hospital were randomly selected. The hospital formed an evaluation team that conducted preliminary evaluations of these prescriptions and an expert team that re-evaluated any problematic prescriptions. We analysed the irrational prescription rate, proportion of antibiotic use, and consistency between the evaluation and expert teams. At the end of the evaluation period, the utilisation rate of single antibiotics in the outpatient and emergency departments increased, the irrational prescription rate decreased, and the proportion of sold antibiotics gradually decreased. In addition, the consistency of prescription evaluation results between the evaluation and expert groups increased over time. In conclusion, monthly evaluation of antibiotic prescriptions is an effective management tool for the rational use of antibiotics in clinical practice and plays an important role in safe clinical drug use.

\section{Introduction}

Since the 1940s, numerous antibiotics have been developed for human use. Antibiotics have saved countless lives in clinical practice and have become one of the most commonly prescribed drugs in human medicine[1]. However, with the increasing use of antibiotics, resistance has substantially increased worldwide[2]. Antibiotic resistance leads to treatment failure, prolonged hospitalisation, and increased medical expenses and mortality[3]. In 2011, the theme for World Health Day was 'Combat Antimicrobial Resistance', which the World Health Organization (WHO) used to call for global attention to the problem of antibiotic resistance[ 4,5$]$. Ten years later, antibiotic resistance remains an urgent public health challenge. Over 2.8 million people are infected with drug-resistant microbes each year in the United States of America alone, resulting in more than 35000 hospitalisations and deaths. The economic and human costs associated with these infections are enormous. By 2050, deaths from drug-resistant infections are expected to rise to 10 million yearly, surpassing diabetes, heart disease, and cancer as the leading cause of human death[6].

The main cause of antibiotic resistance is the inappropriate use of antibiotics. It is necessary to reduce the excessive rate of antibiotic prescription and improve the use of antimicrobials to curb resistance[7]. Performing audits and providing feedback on antibiotic prescriptions have been shown to effectively control antibiotic use and influence clinical outcomes. For example, prospective audits with interventions and feedback can considerably reduce the total number of prescribed antibiotics, as well as improve the quality of antibiotic prescriptions $[8,9]$. Integrating prescription restrictions, approval systems, and computer decision support (CDS) into the electronic prescription process is an effective measure to reduce antibiotic resistance[10-12]. The above-mentioned measures were designed to improve the antibiotic prescription process but are limited by the ability of individual reviewers. However, the effect of periodic retrospective analyses of antibiotic prescriptions on antibiotic use seems to be unexplored. We 
believe that professional evaluation teams may have a more substantial impact on antibiotic management than individual reviewers. Periodic sampling evaluation of antibacterial prescriptions has rarely been reported. This study established a monthly evaluation system, combined with an electronic prescription system, and verified whether the combination of these two systems could improve the rational use of antibacterial drugs. This innovative method involves three rounds of evaluation and publication of results and acts as an important supplement to prospective prescription reviews by improving the efficiency of physicians and evaluators.

\section{Results}

\subsection{Proportion and Number of Antibiotic Prescriptions in Outpatient and Emergency Departments}

After implementing the monthly evaluation system, we found that the utilisation rate of single antibiotic prescriptions in the outpatient department increased yearly from July 2016 to June 2019; the utilisation rate increased by 3.53 and 2.99 percentage points in the outpatient and emergency departments, respectively. In addition, the utilisation rate of combined prescriptions of two antibiotics decreased gradually. However, the rate of antibiotic utilisation in the emergency department was always higher than in the outpatient department each year. In 2018 and 2019, the utilisation rate of triple antibiotic prescriptions was $0.00 \%$ (Table 1 ). 
Table 1

Proportion of antibiotic prescriptions in the outpatient and emergency departments

\begin{tabular}{|c|c|c|c|c|}
\hline \multicolumn{5}{|l|}{ A. Outpatient department } \\
\hline Item & 2016 & 2017 & 2018 & 2019 \\
\hline Number of patients & 169676 & 341774 & 353385 & 368923 \\
\hline Number of antibacterial agents used & 26953 & 56430 & 57615 & 55853 \\
\hline Proportion of antibiotic prescriptions (\%) & $15.88 \%$ & $16.51 \%$ & $16.30 \%$ & $15.14 \%$ \\
\hline Utilisation rate of single antibiotic prescriptions (\%) & $89.70 \%$ & $90.38 \%$ & $90.02 \%$ & 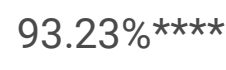 \\
\hline Utilisation rate of two antibiotic prescriptions (\%) & $10.23 \%$ & $9.55 \%$ & $9.98 \%$ & 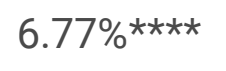 \\
\hline Utilisation rate of triple antibiotic prescriptions (\%) & $0.07 \%$ & $0.07 \%$ & $0.00 \%$ & $0.00 \%$ \\
\hline \multicolumn{5}{|l|}{ B. Emergency department } \\
\hline Item & 2016 & 2017 & 2018 & 2019 \\
\hline Number of patients & 32017 & 64226 & 62886 & 62778 \\
\hline Number of antibacterial agents used & 15708 & 32540 & 31453 & 32519 \\
\hline Proportion of antibiotic prescriptions (\%) & $49.06 \%$ & $50.66 \%$ & $50.02 \%$ & $51.80 \%$ \\
\hline Utilisation rate of single antibiotic prescriptions (\%) & $86.76 \%$ & $85.37 \%$ & $87.45 \%$ & 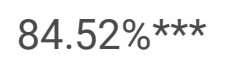 \\
\hline Utilisation rate of two antibiotic prescriptions (\%) & $12.71 \%$ & $14.04 \%$ & $12.54 \%$ & 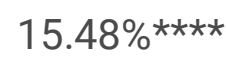 \\
\hline Utilisation rate of triple antibiotic prescriptions (\%) & $0.53 \%$ & $0.59 \%$ & $0.00 \%$ & $0.00 \%$ \\
\hline
\end{tabular}

From an economic perspective, antimicrobial drugs decreased from $16.00 \%$ of total drug sales in 2016 , to $14.73 \%$ in 2019 . However, this difference was not statistically significant and may be due to the proportion of emergency antibiotics used (Table 2). These results suggest that the monthly evaluation system helped to control the number of antibiotic prescriptions. 
Table 2

Number of antibiotic prescriptions in the outpatient and emergency departments

\begin{tabular}{|llll|}
\hline Year & $\begin{array}{l}\text { Total amount of drug } \\
\text { (ten thousand Yuan) }\end{array}$ & $\begin{array}{l}\text { Total amount of antibiotics } \\
\text { (ten thousand Yuan) }\end{array}$ & Percentage \\
\hline 2016 & 2318.4 & 371.01 & $16.00 \%$ \\
\hline 2017 & 4883.52 & 765.81 & $15.68 \%$ \\
\hline 2018 & 5061.06 & 741.03 & $14.64 \%$ \\
\hline 2019 & 5058.64 & 745.5 & $14.74 \%$ \\
\hline Data for 2016 were obtained from July to December & \\
\hline
\end{tabular}

\subsection{Irrational Rate of Antimicrobial Drug Prescription}

An important aspect of the monthly evaluation system was notification of the relevant physician and publication of why the prescription was classified as unreasonable. The effect of this measure was directly reflected in the decrease in proportion of irrational prescriptions. From July 2016 to June 2019, the rate of irrational prescriptions showed a downward trend. The irrationality rate in 2019 decreased by 1.6 percentage points, compared with the irrationality rate from July 2016 to December 2016 (Table 3).

Table 3

Irrational rate of antimicrobial drug prescriptions

\begin{tabular}{|llll|}
\hline Year & Total number of prescriptions evaluated & $\begin{array}{l}\text { Total number of } \\
\text { irrational prescriptions }\end{array}$ & Percentage \\
\hline 2016 & 7397 & 222 & $3 \%$ \\
\hline 2017 & 17908 & 533 & $2.9 \%$ \\
\hline 2018 & 18581 & 427 & $2.27 \%$ \\
\hline 2019 & 18403 & 219 & $1.19 \%^{\star \star \star \star}$ \\
\hline
\end{tabular}

$\star \star \star \star$ indicates that the data were considered statistically significant, $P<0.0001$. Data for 2016 were obtained from July to December.

\subsection{Consistency of Prescription Evaluation Results}

Given the risk of possible misevaluation by the evaluation team, a reassessment system and feedback channel were incorporated into the evaluation system. The number of unqualified prescriptions obtained by each expert group reassessment and the clinical pharmacists' initial evaluations was summarised to determine the consistency of prescription evaluation results for the initial reassessment. The results reveal that the consistency rate between the initial and re-evaluation results increased from 2016 to 2019 (Table 4). 
Table 4

Consistency of prescription evaluation results from the evaluation and the expert teams

$\begin{array}{llll}\text { Year } & \begin{array}{l}\text { Total number of irrational prescriptions in the } \\ \text { preliminary evaluation }\end{array} & \begin{array}{l}\text { Total number of } \\ \text { irrational prescriptions in } \\ \text { the reevaluation }\end{array} & \begin{array}{l}\text { Consistency } \\ (\%)\end{array}\end{array}$

2016350

222

$85.71 \%$

$2017 \quad 601$

533

$88.69 \%$

2018473

427

$90.27 \%$

2019501

480

$95.81 \%^{\star \star \star}$

Consistency of prescription evaluation $(100 \%)=$ Total number of irrational prescriptions in reevaluation/Total number of irrational prescriptions in preliminary evaluation $\times 100$. ${ }^{* \star}$ Indicates that the data were considered statistically significant, $P<0.001$. Data for 2016 were obtained from July to December.

\section{Discussion}

Antibiotics play an important role in modern medicine. The discovery and use of antibiotics have led to medical breakthroughs, such as infection prevention after surgery. Furthermore, antibiotics have made remarkable contributions to the control of infectious diseases[13]. As a result, human life expectancy has increased substantially in the second half of the last century. However, antibiotic resistance is a rapidly growing problem. Resistance to widely used $\beta$-lactam antibiotics has substantially increased, and treatment of ceftriaxone- and fluoroquinolone-resistant gonorrhoea has become a challenge[2]. Moreover, vancomycin, a last line of defence, can no longer completely eradicate Enterococcus faecalis[14]. Although the spread of antibiotic-resistant bacteria threatens human health, research on new antibiotics is slow. For economic reasons, large pharmaceutical companies are turning to the research and development of drugs for chronic diseases[15]; this fact indicates that we are currently entering the postantibiotic era. The loss of antibiotics in clinical treatment implies that common bacterial infections may lead to increased mortality rates in humans.

Fortunately, the link between antibiotic use and resistance has been recognised[16]. Prospective reviews and interventions in antibiotic prescription are considered necessary measures in most healthcare systems. Antibiotic prescription management and monitoring, with the participation of clinical pharmacists, can effectively reduce the use of antibiotics and financial waste[15, 17, 18]. The use of electronic prescriptions has also improved the effectiveness of antibiotic management. In addition, studies have found that different multi-antibiotic management regimens can achieve better control than a single program[19]. However, it is still necessary to explore other ways to manage antibiotic use.

In this study, we established a monthly evaluation system using random sampling and retrospective analysis of antibiotic prescriptions in the outpatient and emergency departments. In contrast with prospective reviews and interventions by clinical pharmacists, the monthly evaluation system responds to 
the execution or review of antibiotic prescriptions. The monthly evaluation system supplements the prospective review and intervention systems. Its main function is to compensate for human negligence in prescription review and, more importantly, to optimise the qualified prescription. This was demonstrated by the change in the proportion of antibiotics prescribed throughout this study, particularly the reduced irrational prescription rate. Although the prescriptions approved by clinical pharmacists are qualified and have good curative effects, there is still room for improvement in the management of antibiotics, owing to the differences in experiences and habits of physicians. In this study, the evaluation team evaluated prescription samples, which were then reviewed by the team leader. As a result of these two rounds of review, the evaluation errors were minimised. The subsequent publishing of the results was one of the key aspects of the system. A study has suggested that the disclosure of institutional assessment scores could eventually change clinical practice[20]. Similarly, our results suggest that disclosing the evaluation results of physicians' antibiotic prescriptions can also improve the level of antibiotic management by physicians and pharmacists. The publication includes unreasonable prescriptions and the evaluation team's suggestions for improvement. This serves as feedback to the relevant physicians and pharmacists and as a warning to other physicians. Any objection to the publication can be reported to the evaluation expert panel by the physician, who can then apply for a re-evaluation. As the final step in our study, the expert group ensured the accuracy of evaluations and improved the operational level of the evaluation team by assessing the annual improvement in the initial response-to-evaluation consistency rate. More importantly, the change in antibiotic use did not affect disease treatment during the time in which the system was implemented. There was no significant change in the rate of patient complaints about treatment outcomes.

However, there were limitations to the system. It did not achieve the same results for antibiotic prescription management in the emergency department as in the outpatient department. There was a decrease in the proportion of single antibiotics prescribed in the emergency department, and there was a significant increase in the proportion of two-dose antibiotics prescribed (Table 1B). These results may be attributed to the fact that most patients in the emergency department are in critical condition and are administered extensive antimicrobial therapy for their survival. In future, we hope to study and improve the antimicrobial-prescribing situation in the emergency department.

In conclusion, we established a monthly evaluation system to assess antibiotic prescriptions, with three rounds of evaluation and a public feedback system. We verified the effectiveness of the system on clinical antibiotic use management and improved the professionalism of medical staff, including evaluation team members. At present, the system is running efficiently. However, owing to the impact of coronavirus disease 2019 in 2020, the number of patients has increased dramatically. This study only intercepted the system data from July 2016 to June 2019 for verification to exclude pandemic-related factors. Detailed research is needed to develop more effective management systems for antibiotic use.

\section{Methods


A total of 58,749 antibiotic prescriptions from the outpatient and emergency departments of the Ningbo Sixth Hospital were randomly selected from July 2016 to June 2019.

\subsection{Basis for Assessment}

The criteria for prescription comment included: Drug description, Prescribing point review management practices (Trial), 2010, China[21]; Scheme of special remediation activity on the clinical application of antimicrobial drugs, 2012, China[22]; Guidelines for clinical use of antimicrobial agents, 2015, China[23]; Clinical guidelines; and UpToDate database (Wolters Kluwer, Alphen aan den Rijn, The Netherlands).

\subsection{Sampling Method}

We first enabled the iPharmacare Drug Management System (IDMS) (iPharmacare, Hangzhou, Zhejiang, China). Every month, $25 \%$ of the doctors in our hospital who were qualified to prescribe antibiotics were randomly selected to evaluate the antibiotics prescribed in the outpatient and emergency departments. Each prescription, having a total number below 50, was evaluated. Additionally, the number of antibiotic prescriptions was at least $25 \%$ of the total number of prescriptions in the hospital.

\subsection{Evaluation Method}

Prescription evaluation groups were established under the leadership of the Medical Services section and comprised evaluation and expert teams. The evaluation team conducted initial evaluations of randomly selected prescriptions, while the expert team conducted re-evaluations of irrational prescriptions.

For the initial evaluation, clinical pharmacists from the evaluation team assessed sample prescriptions, and recorded irrational prescriptions using Microsoft Excel (2010) spreadsheets. Irrational prescription types, problem descriptions, medication suggestions, and other parameters were then analysed.

Next, the prescription evaluation team leader re-evaluated any unreasonable prescriptions, and the hospital intranet publicised the irrational prescription for 1 week. The prescription evaluation team leader was contacted for any issues, and experts were organised to re-evaluate the problem to obtain the final evaluation result.

The evaluation team consisted of six clinical pharmacists, and the expert team consisted of two chief pharmacists and two chief physicians. The sampling and preliminary evaluation of the previous month were completed before the 15th of the next month. The evaluation results were publicised and studied by the whole hospital on the 15th of each month. If there were any objections within 7 days, the expert team would start the re-evaluation; otherwise, the evaluated result remained in effect.

\subsection{Assessment Measures}

Unreasonable prescriptions were publicised regularly, and the final result was included in evaluating individual senior professional titles. For each unqualified prescription, 0.1 points were deducted. 


\subsection{Ethical Approval}

The ethical approval to conduct this retrospective study was obtained from the Ethics Committee of Ningbo Sixth Hospital. All experiments were performed in accordance with relevant guidelines and regulations.

\subsection{Statistical Analysis}

SPSS version 16.0 (IBM, Armonk, NY, USA) was used for data processing, and $\mathrm{P}<0.05$ was considered as statistically significant. The rate of irrational prescriptions of antibacterial drugs in different years was analysed using the chi-square test.

\section{Declarations}

\section{Acknowledgements}

We thank all of our colleagues in the hospital who have been involved in the operation and research of the system. We would like to thank Editage (www.editage.cn) for English language editing.

\section{Author contributions}

Conceptualization, Qiong Yang and Junhong He and Fangfang Yuan; Methodology, Fangfang Yuan and Junhong He; Software, Qiong Yang.; Formal Analysis, Qiong Yang and Junhong He; Investigation, Fangfang Yuan.; Resources, Li Li, Jianfeng Jin,; Data Curation, Qiong Yang and Junhong He; Writing Original Draft Preparation, Qiong Yang, Jnhong He.; Writing - Review \& Editing, Li Li, Jianfeng Jin, Junhong He ; Supervision, Li Li, Jianfeng Jin, Junhong He; Project Administration, Li Li, Jianfeng Jin, Junhong He.; Funding Acquisition, Qiong Yang.

\section{Competing interests}

The authors confirm that there are no conflicts of interest.

\section{Data availability}

All relevant data is contained within the article: The original contributions presented in the study are included in the article/supplementary material, further inquiries can be directed to the corresponding author/s.

\section{References}


1. J. Davies, D. Davies, Origins and evolution of antibiotic resistance, Microbiology and molecular biology reviews : MMBR. 74, 417-33 (2010)

2. J.I. Alós, [Antibiotic resistance: A global crisis], Enfermedades infecciosas y microbiologia clinica. 33, 692-9 (2015)

3. M. Ferri, E. Ranucci, P. Romagnoli, et al., Antimicrobial resistance: A global emerging threat to public health systems, Critical reviews in food science and nutrition. 57, 2857-2876 (2017)

4. H. Goossens, M. Ferech, R. Vander Stichele, et al., Outpatient antibiotic use in Europe and association with resistance: a cross-national database study, Lancet (London, England). 365, 579-87 (2005)

5. I.N. Okeke, R. Laxminarayan, Z.A. Bhutta, et al., Antimicrobial resistance in developing countries. Part I: recent trends and current status, The Lancet. Infectious diseases. 5, 481-93 (2005)

6. L. Morrison, T.R. Zembower, Antimicrobial Resistance, Gastrointestinal endoscopy clinics of North America. 30, 619-635 (2020)

7. E.J. Septimus, Antimicrobial Resistance: An Antimicrobial/Diagnostic Stewardship and Infection Prevention Approach, The Medical clinics of North America. 102, 819-829 (2018)

8. H. Benoist, A. de La Blanchardière, A. Flatres, et al., Effect of a prospective audit with intervention and feedback on Clostridioides difficile infection management, International journal of clinical pharmacy. 42, 923-930 (2020)

9. L.R. Taggart, E. Leung, M.P. Muller, et al., Differential outcome of an antimicrobial stewardship audit and feedback program in two intensive care units: a controlled interrupted time series study, BMC infectious diseases. 15, 480 (2015)

10. K.A. Thursky, K.L. Buising, N. Bak, et al., Reduction of broad-spectrum antibiotic use with computerized decision support in an intensive care unit, International journal for quality in health care : journal of the International Society for Quality in Health Care. 18, 224-31 (2006)

11. R.S. Evans, S.L. Pestotnik, D.C. Classen, et al., A computer-assisted management program for antibiotics and other antiinfective agents, The New England journal of medicine. 338, 232-8 (1998)

12. J.C. McGregor, E. Weekes, G.N. Forrest, et al., Impact of a computerized clinical decision support system on reducing inappropriate antimicrobial use: a randomized controlled trial, Journal of the American Medical Informatics Association : JAMIA. 13, 378-84 (2006)

13. Y. Arakawa, [Epidemiology of drug-resistance and clinical microbiologists in the 21 st century], Rinsho byori. The Japanese journal of clinical pathology. Suppl 111, 1-8 (2000)

14. J. Edelsberg, D. Weycker, R. Barron, et al., Prevalence of antibiotic resistance in US hospitals, Diagnostic microbiology and infectious disease. 78, 255-62 (2014)

15. S.J. Projan, D.M. Shlaes, Antibacterial drug discovery: is it all downhill from here?, Clinical microbiology and infection : the official publication of the European Society of Clinical Microbiology and Infectious Diseases. 10 Suppl 4, 18-22 (2004)

16. B.G. Bell, F. Schellevis, E. Stobberingh, et al., A systematic review and meta-analysis of the effects of antibiotic consumption on antibiotic resistance, BMC infectious diseases. 14, 13 (2014) 
17. V. Nault, J. Pepin, M. Beaudoin, et al., Sustained impact of a computer-assisted antimicrobial stewardship intervention on antimicrobial use and length of stay, The Journal of antimicrobial chemotherapy. 72, 933-940 (2017)

18. D.A. Goff, G.H. Karam, S.T. Haines, Impact of a national antimicrobial stewardship mentoring program: Insights and lessons learned, American journal of health-system pharmacy : AJHP : official journal of the American Society of Health-System Pharmacists. 74, 224-231 (2017)

19. D. Baur, B.P. Gladstone, F. Burkert, et al., Effect of antibiotic stewardship on the incidence of infection and colonisation with antibiotic-resistant bacteria and Clostridium difficile infection: a systematic review and meta-analysis, The Lancet. Infectious diseases. 17, 990-1001 (2017)

20. T. Kim, Y.K. Do, Prescription of Systemic Steroids for Acute Respiratory Infections in Korean Outpatient Settings: Overall Patterns and Effects of the Prescription Appropriateness Evaluation Policy, Journal of preventive medicine and public health = Yebang Uihakhoe chi. 53, 82-88 (2020)

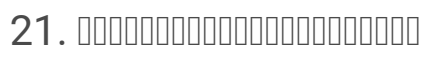

Q,http://www.nhc.gov.cn/wjw/ywfw/201306/094ebc83dddc47b5a4a63ebde7224615.shtml. Published 2010,Accessed February 10th 2010

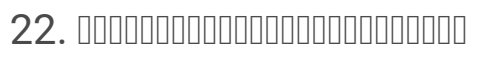

囚,http://www.nhc.gov.cn/wjw/xwdt/201305/e1a64829a869405e9d378191d00d860b.shtml. Published 2013,Accessed May 62013

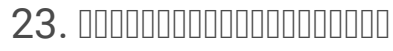

囚,http://www.nhc.gov.cn/wjw/gfxwj/201304/2c850f3dc54244ca846d8a17baf3613d.shtml. Published 2004,Accessed August 19th 2004 